\title{
Efficacy of Afatinib and Lapatinib Against HER2 Gene-amplified Trastuzumab-sensitive and -resistant Human Gastric Cancer Cells
}

\author{
SUSUMU NAKATA ${ }^{1}$, MITSUGU FUJITA ${ }^{2}$ and HAYAO NAKANISHI ${ }^{3}$ \\ ${ }^{1}$ Department of Clinical Oncology, Kyoto Pharmaceutical University, Kyoto, Japan; \\ ${ }^{2}$ Department of Microbiology, Faculty of Medicine, Kindai University, Osaka, Japan; \\ ${ }^{3}$ Department of Gastroenterological Surgery, Graduate School of Medicine, Nagoya University, Nagoya, Japan
}

\begin{abstract}
Background/Aim: Trastuzumab is the only clinically approved targeted therapy for HER2 geneamplified gastric cancer at present. However, the clinical significance of multi-targeting tyrosine kinase inhibitors (TKIs) in HER2-positive gastric cancer remains unclear. Materials and Methods: We examined the anti-tumor activity of lapatinib and afatinib, that are reversible and irreversible TKIs, in HER2 gene-amplified trastuzumab-sensitive andresistant gastric cancer cells (GLM-1 and GLM-1HerR2) in vitro and in vivo. Results: Afatinib inhibited the growth of GLM-1 and GLM-1HerR2 cells in vitro more efficiently than lapatinib by inducing $G_{1}$ cell-cycle arrest and apoptosis. Preclinical studies in mice revealed that afatinib inhibited growth of intraperitoneal GLM-1 and subcutaneous GLM1 HerR2 tumor more strongly than lapatinib. Afatinib was more effective than lapatinib in blocking PI3K/Akt and MAPK signaling in both GLM-1 and GLM-1HerR2 cells. Conclusion: Afatinib could be a potential new moleculartargeted therapy for trastuzumab-sensitive and trastuzumabresistant HER2 gene-amplified gastric cancers.
\end{abstract}

Gastric cancer is the sixth most common malignancy worldwide, and the third leading cause of cancer-related death (1). HER2-overexpressing gastric cancers have been observed at an incidence of approximately $20 \%$ of patients with gastric cancer, and are known to be highly metastatic with a poor prognosis. Trastuzumab is the first therapeutic antibody approved for HER2-positive breast and gastric

Correspondence to: Susumu Nakata, MD, Ph.D., Department of Clinical Oncology, Kyoto Pharmaceutical University, MisasagiNakauchicho 5, Yamashina-ku, Kyoto 607-8414, Japan. Tel: +81 (0)755954719, e-mail: snakata@mb.kyoto-phu.ac.jp

Key Words: Gastric cancer, afatinib, tyrosine kinase inhibitor, HER2, trastuzumab resistance. cancer patients and is still the only clinically approved targeted therapy for HER2-positive gastric cancer (2-3). However, acquired resistance to trastuzumab is unavoidable. Thus, a potential strategy for overcoming such resistance is urgently required.

In patients with trastuzumab-resistant breast cancer, it was shown that treatment with the tyrosine kinase inhibitor (TKI) lapatinib could provide a substantial clinical benefit (4). However, in HER2-positive gastric cancer patients, the TyTAN study, a randomized phase III trial, demonstrated that lapatinib plus chemotherapy did not significantly improve prognosis (5). Afatinib is an irreversible multi-targeting TKI that covalently binds to EGFR, HER2 and HER4. Recently, afatinib was shown to be effective against lung cancer (6), and is currently approved as a first-line therapy (7). In HER2-positive gastric cancer, the anti-tumor efficacy of afatinib was recently demonstrated in preclinical models (89). However, the potential utility of afatinib in trastuzumabresistant gastric cancer remains to be determined.

We previously established $H E R 2$ gene-amplified gastric cancer cell lines (10) and generated a trastuzumab-resistant gastric cancer cell line by in vivo selection (11). In the present study, we evaluated the anti-tumor activity of afatinib compared with lapatinib in trastuzumab-sensitive and trastuzumab-resistant gastric cancer cells.

\section{Materials and Methods}

Compounds. Afatinib was kindly provided by Boehringer Ingelheim GmbH (Ingelheim am Rhein, Germany). Lapatinib was purchased from Santa Cruz (Santa Cruz, CA, USA), trastuzumab from Chugai Pharmaceuticals (Tokyo, Japan), and heregulin from R\&D Systems (Minneapolis, MN, USA). The following antibodies were used: for western blot, total Erk1/2, phospho-Erk1/2 (Thr202/Tyr204), HER2, total Akt, and phospho-Akt (Ser473) (CST, Beverly, MA, USA), HER3 (clone 2F12; Upstate, Lake Placid, NY, USA), and $\beta$-actin (Sigma-Aldrich, St. Louis, MO, USA); for immunohistochemistry, EGFR (Novocastra Labs, Newcastle, UK), HER2 (Dako/Agilent, 
Santa Clara, CA, USA), and HER3 (clone Ab-10; NeoMarkers, Fremont, CA, USA).

Cell culture. The GLM-1 cell line was established in our laboratory from surgically resected liver metastasis of a Japanese gastric cancer patient (10). Trastuzumab-resistant GLM-1 cells (GLM-1HerR2) were isolated after three cycles of in vivo selection with intraperitoneal (i.p.) trastuzumab treatment and subsequent culture of the residual metastatic tumor cells (11). These cells were maintained in DMEM supplemented with $10 \%$ fetal bovine serum, $100 \mathrm{units} / \mathrm{ml}$ penicillin, and $100 \mu \mathrm{g} / \mathrm{ml}$ streptomycin, and were incubated at $37^{\circ} \mathrm{C}$ in $5 \% \mathrm{CO}_{2}$.

Cell growth inhibition assay. Cells were plated at $1 \times 10^{4}$ cells/well in 96-well plates and treated with increasing doses of lapatinib, afatinib $(0,0.1,1.0$, and $10 \mu \mathrm{M})$, or trastuzumab $(0,1,10$, and 100 $\mu \mathrm{g} / \mathrm{ml})$. The number of viable tumor cells was counted on day 3 with a hemocytometer.

Cell cycle analysis. The procedure has been previously described (11). Briefly, cells were treated with lapatinib and afatinib for $24 \mathrm{~h}$. Then, DNA was stained with propidium iodide (PI) using the CycleTEST Plus Kit (Becton Dickinson, San Jose, CA, USA), and analyzed with a FACSCalibur instrument (Becton Dickinson Biosciences, San Diego, CA, USA), and the ModFit software (Topsham, ME, USA).

Apoptosis assay. Briefly, cells were plated at $1 \times 10^{5}$ cells/condition in 6-cm plates and treated with lapatinib or afatinib $(10 \mu \mathrm{M})$ for $36-$ $48 \mathrm{~h}$, and induction of apoptosis was assessed using the Annexin VFITC Apoptosis Detection Kit (Biovision, CA, USA) according to the manufacturer's instructions.

Western blot analysis. Cells were incubated in serum-free medium for $24 \mathrm{~h}$, exposed to indicated concentrations of the drugs for $2 \mathrm{~h}$ at $37^{\circ} \mathrm{C}$, and then further exposed to heregulin $(25 \mathrm{ng} / \mathrm{ml})$ for $10 \mathrm{~min}$. Whole cell lysates $(50 \mu \mathrm{g})$ were separated by SDS-PAGE, transferred to PVDF membranes, and visualized using Super Signal West Pico chemiluminescence substrate (Thermo Fisher Scientific, Waltham, MA, USA) as previously described (11).

Immunohistochemical and FISH analysis. Immunohistochemistry and FISH analysis were performed as previously described (11). Briefly, sections of subcutaneous (s.c.) tumors were analyzed by standard immunohistochemical staining using the Vectastain ABC kit (Vector Laboratories, Burlingame, CA, USA). EGFR and HER2 expression were scored based on the membrane staining, according to the criteria of the HercepTest. Gene amplification of HER2 in GLM-1 cells was determined by dual-color FISH using the PashVysion HER2 DNA probe kit (Vysis/Abott Molecular, Des Plaines, IL, USA).

Tumor xenograft studies. Five million cells were injected subcutaneously in 8-week-old male nude mice (Shizuoka Laboratory Animal Center, Hamamatsu, Japan). Mice $(n=5)$ were orally administered lapatinib or afatinib at a sub-maximal tolerated dose (SMTD) of 150 or $25 \mathrm{mg} / \mathrm{kg} / \mathrm{day}$, respectively, 4-5 times a week for 4 weeks starting at 1-2 weeks post-injection. Mice were treated with trastuzumab by intraperitoneal injection at a dose of $20 \mathrm{mg} / \mathrm{kg} / \mathrm{day}$, twice a week for 4 weeks. The tumor volume was estimated by the following formula: $\mathrm{L} \times \mathrm{W} \times \mathrm{W} \times 0.5$. In the peritoneal metastasis experiment, mice were inoculated with $1 \times 10^{7}$ luciferase-expressing GLM-1 cells, and monitored using an Ivis Lumina II imager (Caliper Life Science, Hopkinton, MA, USA). After 4 weeks of treatment, the metastatic nodules were collected and weighed. All experiments were carried out with the approval of the Institutional Ethical Committee for Animal Experimentation.

Statistical analysis. The statistical significance of differences between groups was determined by Student's $t$-test using the microsoft Excel software. A $p$-value of $<0.05$ was considered significant.

\section{Results}

Effect of TKIs on HER2-overexpressing GLM-1 cells. Amplification of the HER2 gene and its overexpression in GLM-1 cells were confirmed by FISH and immunohistochemistry (3+) (Figure 1A, left). The growth of GLM-1 cells was strongly inhibited by afatinib and lapatinib, with halfmaximal inhibitory concentrations $\left(\mathrm{IC}_{50}\right.$ ) of 0.2 and $4 \mu \mathrm{M}$, respectively (Figure 1A, right). Cell cycle analysis of GLM-1 cells showed that treatment with lapatinib or afatinib $(10 \mu \mathrm{M})$ resulted in a similar decrease in the percentage of cells in the S-phase and an increase in $\mathrm{G}_{1}$-phase cells (Figure 1B). Annexin $\mathrm{V}$ staining demonstrated that the percentage of early apoptotic GLM-1 cells was more evidently increased by afatinib than lapatinib (Figure 1C). Western blot analysis revealed that afatinib reduced the phosphorylation of EGFR, HER2, and HER3 and their downstream signaling molecules Akt and Erk1/2 more strongly than lapatinib (Figure 1D).

Anti-tumor effects of TKIs alone and in combination with trastuzumab on HER2-positive and-negative cell lines in vivo. The anti-tumor effects of the TKIs (lapatinib and afatinib) were significantly stronger than those of trastuzumab in mice bearing s.c. GLM-1 tumors, and afatinib was slightly more efficacious than lapatinib (Figure 2A). Trastuzumab and lapatinib were well-tolerated in tumorbearing mice, while with afatinib body weight loss (less than $10 \%$ ) was observed (Figure 2B). The anti-metastatic activities of trastuzumab and afatinib were equivalent and greater than those of lapatinib (Figure 2C).

Growth inhibition of trastuzumab-resistant cells (GLM1HerR2) by afatinib and lapatinib. The effects of afatinib and lapatinib on trastuzumab-resistant gastric cancer cells (GLM-1HerR2) were next examined. GLM1-HerR2 cells were resistant to trastuzumab, but their growth was significantly inhibited by afatinib and lapatinib, with $\mathrm{IC}_{50}$ values of 0.1 and $4 \mu \mathrm{M}$, respectively (Figure 3B). Cell cycle analysis revealed comparable $G_{1}$ cell cycle arrest. Annexin $\mathrm{V}$ staining demonstrated that afatinib induced slightly higher levels of apoptosis than lapatinib (Figure 3A). Western blot analyses demonstrated that lapatinib and afatinib comparably 

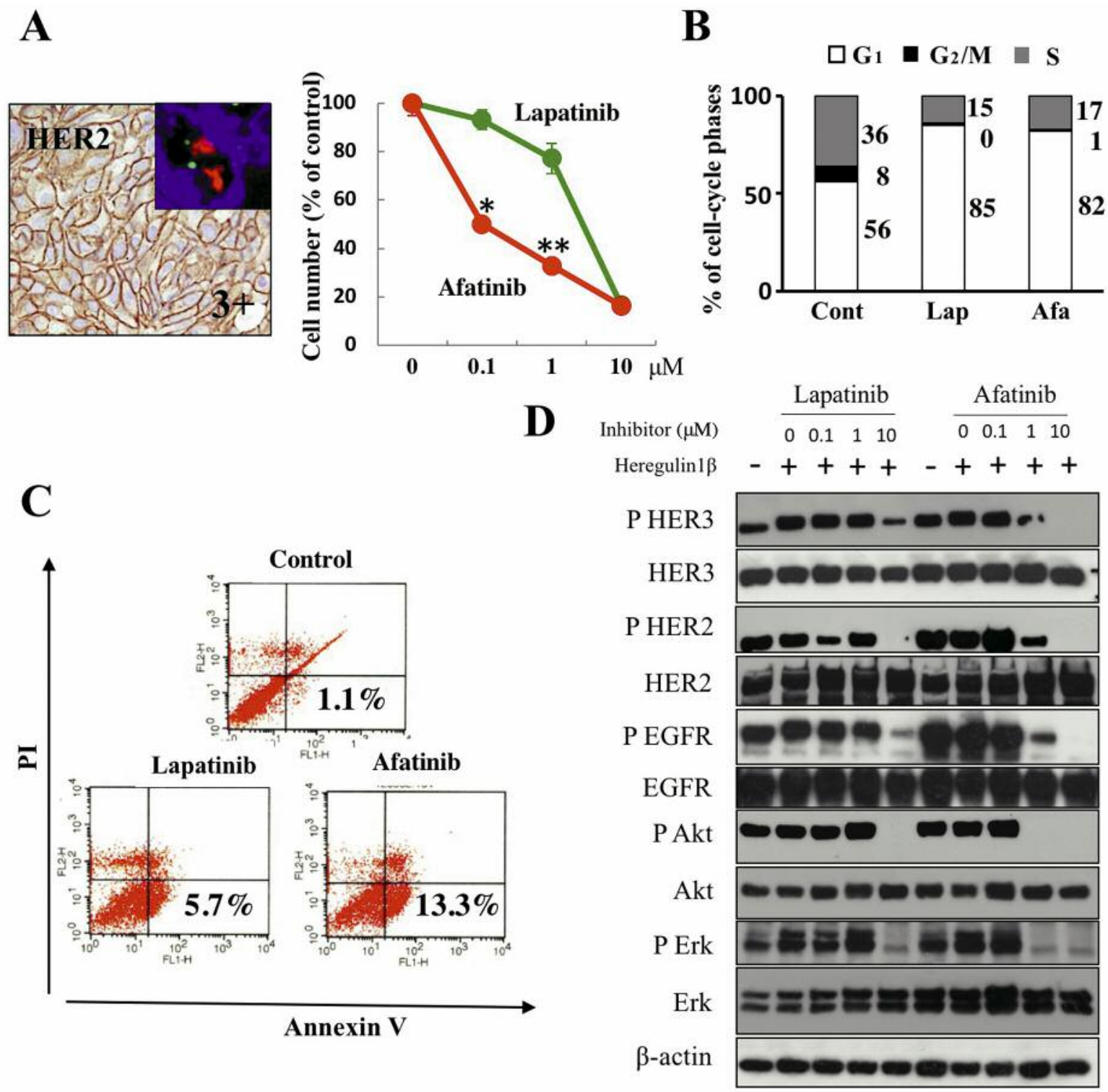

Figure 1. Growth inhibition of GLM-1 cells by afatinib and lapatinib in vitro. A: Immunohistochemical analysis of HER2 expression in GLM-1 xenografts. Inset: HER2 gene amplification detected by FISH. Comparison of growth inhibition of GLM-1 cells by lapatinib and afatinib. *p<0.01, ${ }^{* *} p<0.001$ (vs. lapatinib). Bars: standard error (SE). B: Quantification of the cell-cycle fractions is shown. C: Annexin V assay. The percentage of cells in the right lower quadrant (annexin V+/PI- cells) are shown as early apoptotic cells. D: Western blot analysis of EGFR, HER2 and HER3, and their downstream signaling mediators Akt and Erk in GLM-1cells.

suppressed the phosphorylation of EGFR/HER2/HER3, but afatinib inhibited downstream Akt and Erk1/2 more strongly than lapatinib (Figure 3C). GLM-1HerR2 s.c. tumors were refractory to trastuzumab but were significantly inhibited by TKIs. Afatinib was significantly more effective at inhibiting tumor growth than lapatinib. The combination of these TKIs with trastuzumab exhibited an additive anti-tumor effect on GLM-1HerR2 tumors in mice (Figure 3D).

\section{Discussion}

We previously developed a HER2 gene-amplified trastuzumab-resistant cell line (GLM-1HerR2) by in vivo selection and demonstrated its sensitivity to lapatinib (11). In the present study, we examined afatinib, an irreversible multi-targeted TKI that binds to the kinase domains of EGFR, HER2, and HER4, and found that afatinib had more 
A

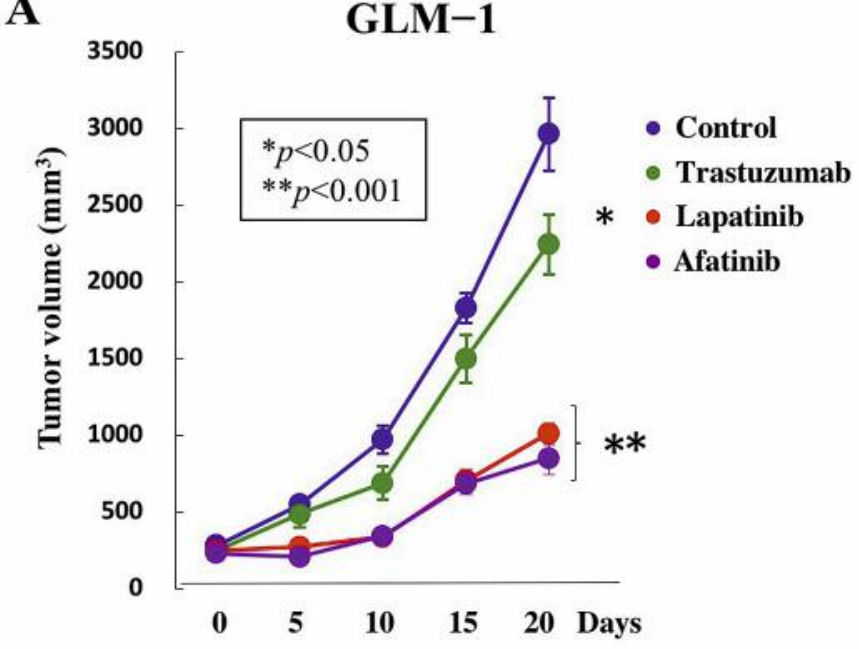

C GLM-1
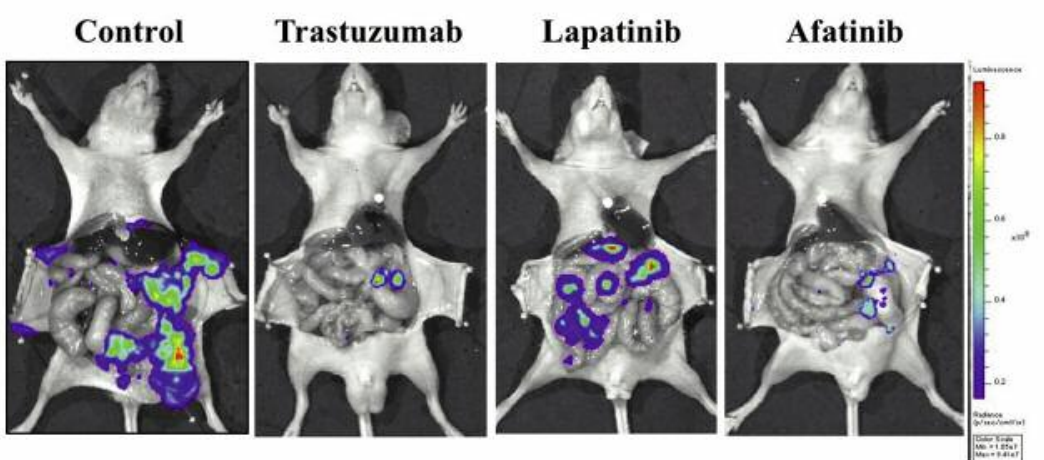

B

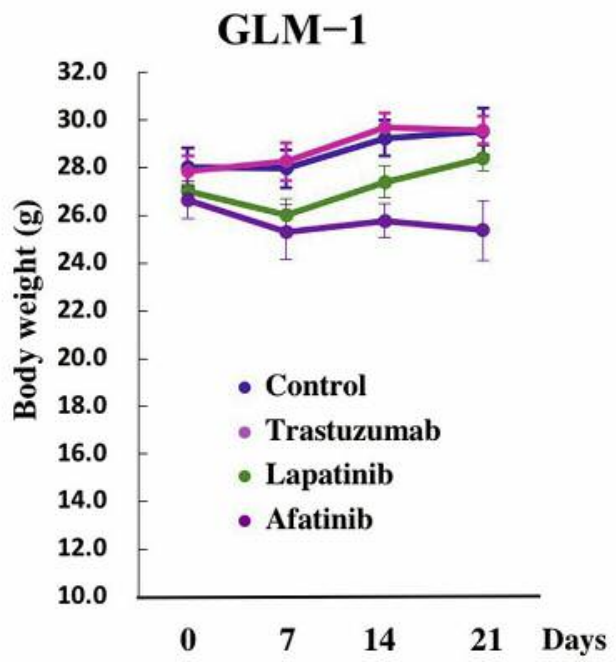

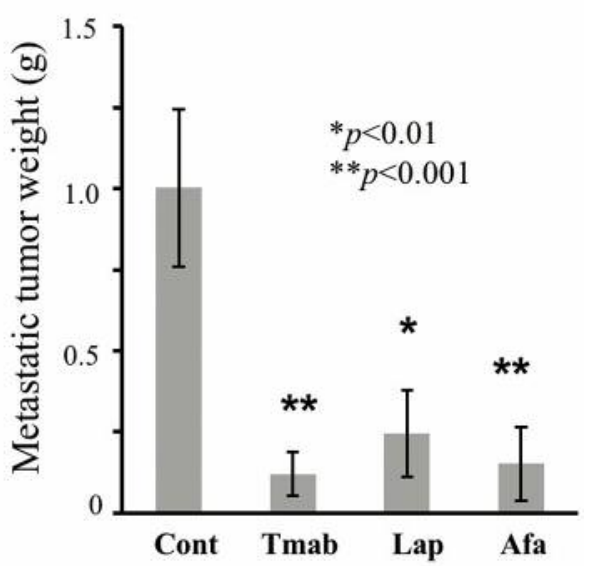

Figure 2. Anti-tumor and anti-metastatic effects of lapatinib, afatinib, and trastuzumab on GLM-1 tumors. A: Effects on the growth of s.c. GLM-1 xenografts in nude mice. ${ }^{*} p<0.05$, ${ }^{*} p<0.001$ (vs. control). Bars: SE. B: Body weight changes in nude mice. C: Effects on GLM-1 peritoneal metastasis. Representative images of peritoneal metastases (left). Quantification of the anti-metastatic effects of the drugs (right). *p<0.01, $* * p<0.001$ (vs. control). Bars: $S E$.

potent anti-tumor activity than lapatinib. A clinical trial of afatinib in patients with metastatic breast cancer resistant to trastuzumab has shown promising results (12). Although two investigators recently reported the efficacy of afatinib against HER2 gene-amplified gastric cancer in preclinical studies, they did not examine the effects of afatinib on trastuzumabresistant cells $(8,9)$, and a phase II clinical study of afatinib in HER2-positive gastric cancer patients is now underway (13). Therefore, to the best of our knowledge, this is the first study demonstrating the potent anti-tumor efficacy of afatinib against $H E R 2$ gene-amplified, trastuzumab-resistant gastric cancer.

Acquired resistance to trastuzumab is the most crucial problem in the treatment of HER2-positive gastric cancer in clinical practice (11). In this study, afatinib inhibited the growth of both GLM-1 and GLM-1HerR2 cells at a much lower concentration than lapatinib in vitro. Furthermore, a preclinical study comparing the anti-tumor activities of afatinib and lapatinib demonstrated that afatinib showed slightly to moderately stronger anti-tumor and anti-metastatic effects on GLM-1 and GLM-1HerR2 tumors than lapatinib, which was associated with stronger inhibition of the PI3K/Akt and MAPK signaling pathways by afatinib. Nonagase et al. reported that elevated PI3K/Akt and/or MAPK signaling led to trastuzumab/lapatinib resistance (14). These results suggest that more potent blockade of PI3K/Akt and MAPK signaling by afatinib due to multiple and irreversible targeting would be critical for the superior anti-tumor activity.

Another interesting finding of this study is that TKIs in combination with trastuzumab showed more potent anti-tumor 


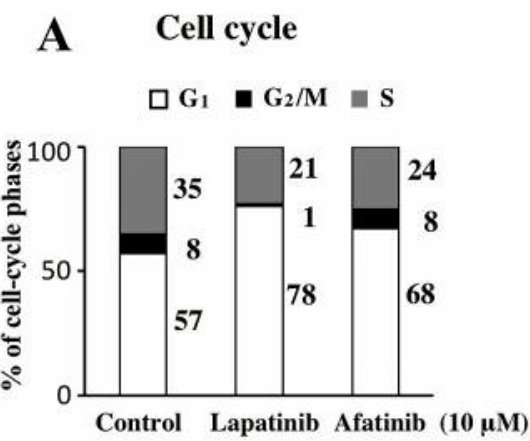

\section{B}

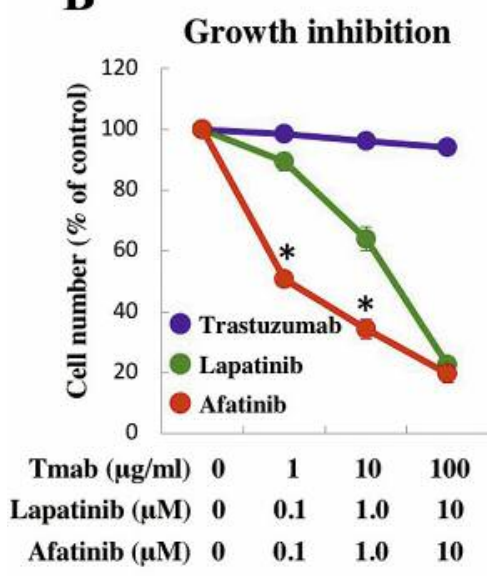

Apoptosis

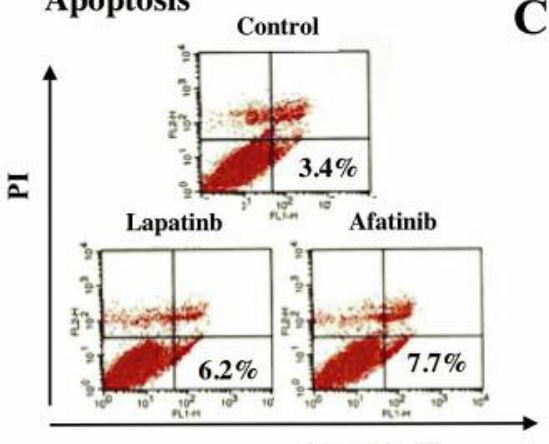

D

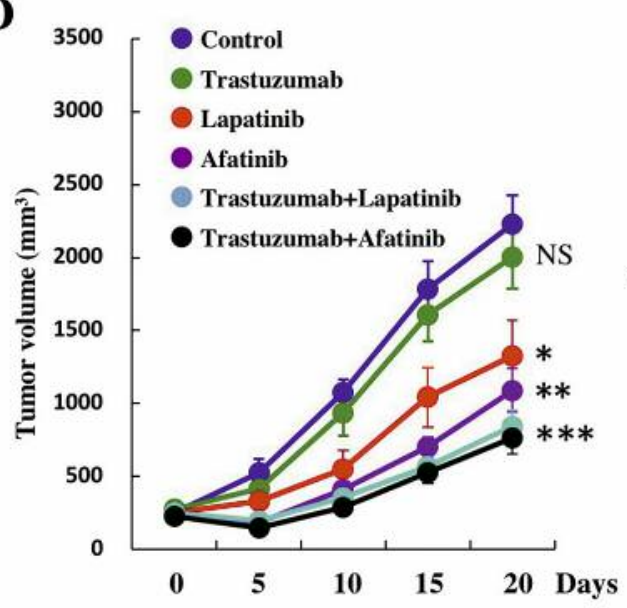

$\mathrm{C}$

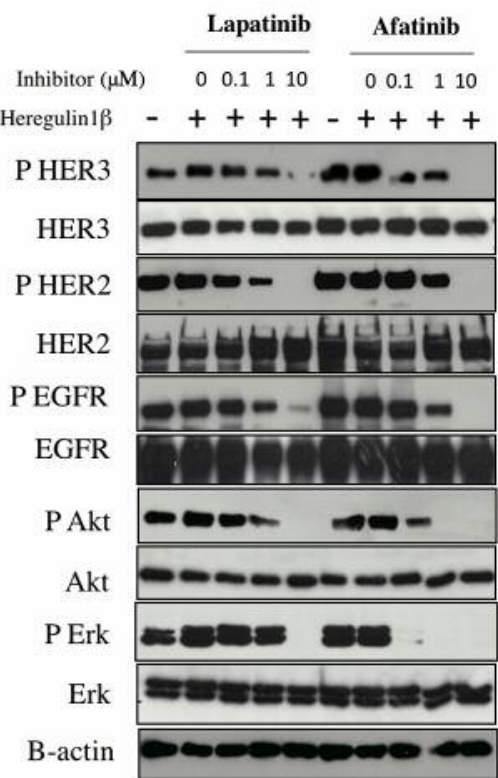

Figure 3. Growth inhibition of trastuzumab-resistant cells (GLM-1HerR2) by lapatinib and afatinib in vitro and in vivo. A: Cell-cycle and apoptotic analysis of GLM1-HerR2 cells. Quantification of the cell-cycle fractions (left). Quantification of apoptosis (right). B: Growth inhibition of GLM1 HerR2 cells in vitro. ${ }^{*} p<0.01$ (afatinib vs lapatinib). Bars: SE. C: Western blot analysis of EGFR, HER2, HER3, Akt and Erk1/2 in GLM-1HerR2 cells treated with lapatinib or afatinib in vitro. D: Anti-tumor effects of lapatinib and afatinib alone and in combination with trastuzumab in sc tumor-bearing mice. $* p<0.05, * * p<0.01, * * * p<0.001$ (vs. control). NS: Not significant. Bars: SE.

activity than the TKIs alone. Consistent with this, Nam et al. have previously reported that the pan-HER inhibitor PF00299804 synergizes with trastuzumab for inhibition of gastric cancer cell growth (15). Spector et al. have also reported that antibody-dependent cellular cytotoxicity (ADCC) could be a potential explanation for the additional benefit of the combination treatment (16). These findings suggest that afatinib in combination with trastuzumab could be a promising strategy for HER2-positive, trastuzumab-resistant gastric cancers.

In conclusion, afatinib had a greater anti-tumor and antimetastatic activity than lapatinib. However, it is of note that afatinib is associated with a manageable, but significant sideeffect, particularly body weight loss mainly due to diarrhea. Nevertheless, afatinib, alone and in combination with trastuzumab, could be an effective therapeutic strategy for patients with trastuzumab-resistant gastric cancer and should, therefore, be further explored in additional preclinical and clinical studies.

\section{Conflicts of Interest}

This study was supported in part by a research grant from Boehringer Ingelheim GmbH (Ingelheim am Rhein, Germany).

\section{Acknowledgements}

The Authors thank Ms. M. Yoshimura and Ms. K. Nishida for their expert technical assistance.

\section{Authors' Contributions}

$\mathrm{SN}$, and $\mathrm{HN}$ performed experiments and drafted the manuscript. MF and $\mathrm{HN}$ designed the experiments, supervised the study, and wrote the manuscript.

\section{References}

1 Bray F, Ferlay J, Soerjomataram I, Siegel RL, Torre LA and Jemal A: Global cancer statistics 2018: GLOBOCAN estimates 
of incidence and mortality worldwide for 36 cancers in 185 countries. CA Cancer J Clin 68: 394-424, 2018. PMID: 30207593. DOI: $10.3322 /$ caac. 21492

2 Wadhwa R, Song S, Lee JS, Yao Y, Wei Q and Ajani JA: Gastric cancer-molecular and clinical dimensions. Nat Rev Clin Oncol 10: 643-655, 2013. PMID: 24061039. DOI: 10.1038/nrclinonc. 2013.170

3 Endo S, Kurokawa Y, Gamoh M, Kimura Y, Matsuyama J, Taniguchi H, Takeno A, Kawabata R, Kawada J, Masuzawa T, Yamamoto K, Kobayashi K, Sakai D, Shimokawa T and Satoh T: Trastuzumab with S-1 plus cisplatin in HER2-positive advanced gastric cancer without measurable lesions: OGSG 1202. Anticancer Res 39: 1059-1065, 2019. PMID: 30711995. DOI: 10.21873/anticanres.13213

4 Blackwell KL, Burstein HJ, Storniolo AM, Rugo H, Sledge G, Koehler M, Ellis C, Casey M, Vukelja S, Bischoff J, Baselga J and O'Shaughnessy J: Randomized study of Lapatinib alone or in combination with trastuzumab in women with ErbB2-positive, trastuzumab-refractory metastatic breast cancer. J Clin Oncol 28 : 1124-1130, 2010. PMID: 20124187. DOI: 10.1200/JCO.2008. 21.4437

5 Satoh T, Xu RH, Chung HC, Sun GP, Doi T, Xu JM, Tsuji A, Omuro Y, Li J, Wang JW, Miwa H, Qin SK, Chung IJ, Yeh KH, Feng JF, Mukaiyama A, Kobayashi M, Ohtsu A and Bang YJ: Lapatinib plus paclitaxel versus paclitaxel alone in the secondline treatment of HER2-amplified advanced gastric cancer in asian populations: TyTAN-A randomized, phase III study. J Clin Oncol 32: 2039-2049, 2014. PMID: 24868024. DOI: 10.1200/ JCO.2013.53.6136

6 Spicer JF and Rudman SM: EGFR inhibitors in non-small cell lung cancer (NSCLC): the emerging role of the dual irreversible EGFR/HER2 inhibitor BIBW 2992. Target Oncol 5: 245-255, 2010. PMID: 20574858. DOI: 10.1007/s11523-010-0140-y

7 Ellis PM, Coakley N, Feld R, Kuruvilla S and Ung YC: Use of the epidermal growth factor receptor inhibitors gefitinib, erlotinib, afatinib, dacomitinib, and icotinib in the treatment of non-small-cell lung cancer: a systematic review. Curr Oncol 22: e183-215, 2015. PMID: 26089730. DOI: 10.3747/co.22.2566

8 Chen Z, Huang W, Tian T, Zang W, Wang J, Liu Z, Li Z, Lai Y, Jiang Z, Gao J and Shen L: Characterization and validation of potential therapeutic targets based on the molecular signature of patient-derived xenografts in gastric cancer. J Hematol Oncol 11: 20, 2018. PMID: 29433585. DOI: 10.1186/s13045-018-0563-y

9 Yoshioka T, Shien K, Namba K, Torigoe H, Sato H, Tomida S, Yamamoto H, Asano H, Soh J, Tsukuda K, Nagasaka T, Fujiwara $\mathrm{T}$ and Toyooka S: Anti-tumor activity of pan-HER inhibitors in HER2-positive gastric cancer. Cancer Sci 109: 1166-1176, 2018. PMID: 29465762. DOI: 10.1111/cas.13546
10 Yokoyama H, Ikehara Y, Kodera Y, Ikehara S, Yatabe Y, Mochizuki Y, Koike M, Fujiwara M, Nakao A, Tatematsu M and Nakanishi $\mathrm{H}$ : Molecular basis for sensitivity and acquired resistance to gefitinib in HER2-overexpressing human gastric cancer cell lines derived from liver metastasis. Br J Cancer 95: 1504-1513, 2006. PMID: 17088902. DOI: 10.1038/sj.bjc.6603459

11 Oshima Y, Tanaka H, Murakami H, Ito Y, Furuya T, Kondo E, Kodera $\mathrm{Y}$ and Nakanishi H: Lapatinib sensitivities of two novel trastuzumab-resistant HER2 gene-amplified gastric cancer cell lines. Gastric Cancer 17: 450-462, 2014. PMID: 23948998. DOI: 10.1007/s10120-013-0290-6

12 Lin NU, Winer EP, Wheatley D, Carey LA, Houston S, Mendelson D, Munster P, Frakes L, Kelly S, Garcia AA, Cleator $\mathrm{S}$, Uttenreuther-Fischer $\mathrm{M}$, Jones $\mathrm{H}$, Wind $\mathrm{S}$, Vinisko $\mathrm{R}$ and Hickish T: A phase II study of afatinib (BIBW 2992), an irreversible ErbB family blocker, in patients with HER2-positive metastatic breast cancer progressing after trastuzumab. Breast Cancer Res Treat 133: 1057-1065, 2012. PMID: 22418700. DOI: 10.1007/s10549-012-2003-y

13 NCT01522768. A phase II study of afatinib and paclitaxel in patients with advanced HER2-positive trastuzumab refractory advanced esophagogastric cancer, 2012. Available at: https://clinicaltrials.gov/ct2/show/NCT01522768

14 Nonagase Y, Yonesaka K, Kawakami H, Watanabe S, Haratani K, Takahama T, Takegawa N, Ueda H, Tanizaki J, Hayashi H, Yoshida T, Takeda M, Chiba Y, Tamura T, Nakagawa K and Tsurutani J: Heregulin-expressing HER2-positive breast and gastric cancer exhibited heterogenous susceptibility to the anti-HER2 agents , lapatinib, trastuzumab and T-DM1. Oncotarget 7: 84860-84871, 2016. PMID: 27768588. DOI: 10.18632/oncotarget.12743

15 Nam HJ, Ching KA, Kan J, Kim HP, Han SW, Im SA, Kim TY, Christensen JG, Oh DY and Bang YJ: Evaluation of the antitumor effects and mechanisms of PF00299804, a pan-HER inhibitor, alone or in combination with chemotherapy or targeted agents in gastric cancer. Mol Cancer Ther 11: 439-451, 2012. PMID: 22135232. DOI: 10.1158/1535-7163.MCT-11-0494

16 Spector NL and Blackwell KL: Understanding the mechanisms behind trastuzumab therapy for human epidermal growth factor receptor 2-positive breast cancer. J Clin Oncol 27: 5838-5847, 2009. PMID: 19884552. DOI: 10.1200/JCO.2009.22.1507

Received September 23, 2019

Revised October 5, 2019

Accepted October 8, 2019 